\title{
Zur Messung des Einflusses von „Augmented Reality“ auf die individuelle Produktivität bei Montagearbeiten
}

\author{
Manfred Rosenberger • Michael Fellmann • Fabienne Lambusch • \\ Michael Poppe $\cdot$ Michael Spitzer
}

Eingegangen: 6. Januar 2020 / Angenommen: 19. April 2020 / Online publiziert: 11. Mai 2020

(C) Der/die Autor(en) 2020

Zusammenfassung Durch die Verfügbarkeit und Leistungsfähigkeit neuartiger Hardware sind zunehmend die Voraussetzungen gegeben, Technologien der erweiterten Realität (Augmented Reality) in der Montage, bzw. dem Zusammenbau von Produkten einzusetzen. Dabei handelt es sich beispielsweise um in das Sichtfeld eingeblendete 3D-Explosionszeichnungen oder unterstützende Zusatzinformationen. Auch wenn ein zentrales Versprechen solcher Ansätze die Erleichterung und Beschleunigung komplexer Montageaufgaben ist, bleibt bisher jedoch weitgehend unklar, welchen Effekt der Einsatz der erweiterten Realität auf die Mitarbeitenden und deren Produktivität tatsächlich hat und wie diese Auswirkungen messbar sind. Für Unternehmen (nicht nur) in Ländern mit hohen Lohnkosten ist eine Beurteilung neuer Technologien hinsichtlich des Einflusses auf die Produktivität allerdings unerlässlich, wobei zudem die Perspektive der Mitarbeitenden bzw. Anwendenden oft vernachlässigt wird. Daher steht in diesem Beitrag die Betrachtung der individuellen Produktivität aus der Perspektive prospektiver Technologieanwendender im Vordergrund. In einem Experten-Workshop wurde dazu ein konkretes Szenario zur Monta-

M. Rosenberger · M. Spitzer

Virtual Vehicle Research GmbH, Graz, Österreich

M. Rosenberger

E-Mail: manfred.rosenberger@v2c2.at

M. Spitzer

E-Mail: michael.spitzer@v2c2.at

M. Fellmann $(\bowtie) \cdot$ F. Lambusch $\cdot$ M. Poppe

Juniorprofessur für Wirtschaftsinformatik, Universität Rostock, Rostock, Deutschland

E-Mail: michael.fellmann@uni-rostock.de

F. Lambusch

E-Mail: fabienne.lambusch@uni-rostock.de

M. Poppe

E-Mail: michael.poppe@uni-rostock.de 
ge eines Getriebes unter Einsatz einer Microsoft HoloLens einer kritischen Analyse unterzogen. Der Beitrag reflektiert anhand dieses Beispiels die Auswirkungen des Technologieeinsatzes und beleuchtet zudem die Akzeptanz von Messverfahren bezüglich der Produktivität. Dazu werden die im Workshop erarbeiteten Wirkfaktoren und Rahmenbedingungen der Messung auch Erkenntnissen aus der Literatur zur Messung von Produktivität gegenübergestellt und die Möglichkeiten und Grenzen der Produktivitätsmessung aufgezeigt.

Schlüsselwörter Produktivitätsmessung · Produktivitätssteigerung ·

Technologieanwender $\cdot$ Augmented Reality $\cdot$ Montage $\cdot$ IT-Unterstützung

\title{
On measuring the influence of "Augmented Reality" on individual productivity in assembly work
}

\begin{abstract}
Due to the availability and performance of novel hardware, the prerequisites are increasingly given for the use of augmented reality technologies in the assembly of products. These technologies include, for example, 3D exploded drawings superimposed in the field of view or supporting additional information. Even though a central promise of such approaches is the facilitation and acceleration of complex assembly tasks, it remains largely unclear what effect the use of augmented reality actually has on employees and their productivity and how these effects can be measured. For companies (not only) in countries with high labor costs, however, an assessment of new technologies with regard to their impact on productivity is indispensable, and the perspective of the employees or users is often neglected. Therefore, this paper focuses on the consideration of individual productivity from the perspective of prospective technology users. In an expert workshop, a concrete scenario for the assembly of a gearbox using a Microsoft HoloLens was subjected to a critical analysis. Based on this example, the article reflects on the effects of the use of technology and also illuminates the acceptance of measurement methods with regard to productivity. To this end, the impact factors and general conditions of measurement developed in the workshop are compared with findings from the literature on productivity measurement and the possibilities and limits of productivity measurement are pointed out.
\end{abstract}

Keywords Productivity measurement - Productivity increase · Technology users · Augmented reality $\cdot$ Assembly $\cdot$ IT support

\section{Motivation}

Die Einführung neuer Methoden und Werkzeuge in Arbeitsprozessen, z. B. der Einsatz von Technologien der erweiterten Realität (Augmented Reality) in der Montage, führt zu einer Veränderung dieser Prozesse. Dadurch eröffnen sich zwar neue Möglichkeiten, jedoch werden gleichzeitig auch veränderte Anforderungen an die Arbeitenden gestellt. Im Fall der Montage können beispielsweise mittels einer Augmented-Reality-Brille 3D-Explosionszeichnungen oder unterstützende Zusatzinformatio- 
nen in das Sichtfeld eingeblendet werden. Solche Ansätze versprechen komplexe Montageaufgaben zu erleichtern und zu beschleunigen. Während kurzfristige und leicht quantifizierbare Auswirkungen, z.B. auf die Geschwindigkeit der Durchführung von Aufgaben, relativ leicht zu überprüfen sind, ist bisher weitgehend unklar, wie sich derartige Veränderungen der Arbeitsprozesse langfristig auf die individuelle Produktivität der Mitarbeitenden auswirken und wie diese Auswirkungen messbar sind. Unter „Produktivität“ wird dabei - dem klassischen Produktivitätsverständnis der Betriebswirtschaft folgend (Wöhe et al. 2013) - das Verhältnis von realem Output zu realem Input verstanden. Der Begriff ist ursprünglich durch die Produktion geprägt, wodurch die Quantität von Input und Output betont wurde. Mit der Zunahme wissensintensiver Arbeiten spielt bezüglich des Outputs die Qualität aber eine mindestens genauso große Rolle wie die Quantität (Drucker 1999), auch da für manche Arbeiten kaum noch quantitative Maßstäbe festgelegt werden können. Während die Arbeitsgeschwindigkeit insbesondere durch neue Technologien gesteigert werden konnte, rücken inzwischen bei der Betrachtung der Produktivität mitarbeiterzentrierte Ansätze immer weiter in den Fokus, denn wie produktiv Mitarbeitende sind, kann durch eine Reihe von individuellen Faktoren beeinflusst werden (vgl. Abschn. 3). Diese Wirkfaktoren auf die Produktivität können körperlicher Natur sein, z. B. arbeitsbedingte Körperhaltung, oder auch mentaler Natur, z. B. Unterbrechungen bei der Arbeit. Insbesondere sind auch langfristige Effekte in die Betrachtung der Produktivität einzubeziehen, da beispielsweise dauerhafter Stress zu psychischen Krankheiten und damit zum Arbeitsausfall führen kann (Scharnhorst 2012). So ist es für Unternehmen wichtig, die Einflussfaktoren auf die individuelle Produktivität identifizieren zu können, um die Mitarbeitenden dabei zu unterstützen, Herausforderungen bei der Arbeit besser zu bewältigen und die Produktivität positiv zu beeinflussen. Bei einer Veränderung von Arbeitsprozessen sollte eine Evaluierung demnach neue Systeme und Technologien ganzheitlich betrachten. Dabei sollten vor allem auch die Be- und Entlastung von Mitarbeitenden und deren Eindrücke und Erfahrungen berücksichtigt werden, da es nicht zuletzt oft die Mitarbeitenden sind, die über den Erfolg einer neuen Technologie entscheiden und denen in Zeiten des Fachkräftemangels und des „War for Talent“ eine hohe Bedeutung zukommt.

Zur umfassenden Betrachtung produktivitätsrelevanter Parameter ist es durch den sinkenden Platzbedarf moderner Technik möglich, verschiedene in kleinen Geräten wie Uhren und Stirnbändern verbaute Sensoren zu nutzen, die eine Aufzeichnung unterschiedlicher Daten ermöglichen und Nutzende dabei nicht stören oder beeinflussen. Diese Daten zu Parametern wie Herzratenvariabilität (HRV), elektrischer Hautleitwert und physische Aktivität lassen Rückschlüsse auf verschiedene persönliche Wirkfaktoren auf die Produktivität wie kognitive Performance (Tsunoda et al. 2017) oder Stress zu (Rodrigues et al. 2018). Während die wissenschaftliche Literatur bereits verschiedene Ansätze vorschlägt, um die Produktivität beeinflussende Faktoren zu identifizieren und zu messen, sind die Messverfahren für den praktischen Einsatz durch Organisationen einer kritischen Analyse zu unterziehen. In diesem Beitrag wird dabei die Perspektive der einzelnen betroffenen Mitarbeitenden betrachtet. In einem Workshop, an dem zehn Fachkundige aus unterschiedlichen Unternehmensbereichen und Unternehmen teilnahmen, wurde exemplarisch der $\mathrm{Zu}$ - 
sammenbau eines Planetenradgetriebes aus LEGO ${ }^{\circledR 1}$ Technic Bausteinen erprobt, der durch die AR-Brille Microsoft HoloLens unterstützt wurde. Die Teilnehmenden des Workshops sollten dann über ihre Erfahrungen im Umgang mit der neuen Technologie reflektieren und über die Potentiale und Herausforderungen bezüglich der individuellen Produktivität diskutieren. Dieser Beitrag präsentiert die diskutierten positiven und negativen Auswirkungen des Technologieeinsatzes sowie Anmerkungen zur Akzeptanz von Messverfahren bezüglich der individuellen Produktivität.

In Abschn. 2 werden daher zunächst Hintergrundinformationen zum Einsatz von AR-Assistenzsystemen dargestellt, während in Abschn. 3 wissenschaftliche Ansätze zur Messung von Wirkfaktoren auf die Produktivität in Form eines Literaturüberblicks vorgestellt werden. Abschn. 4 präsentiert das im Workshop verwendete Szenario und die Umsetzung der Analyse durch die Workshop-Teilnehmenden. Schließlich werden in Abschn. 5 die im Workshop erarbeiteten Wirkfaktoren und Rahmenbedingungen der Messung den aus der Literatur gewonnenen Erkenntnissen zur Messung von Produktivität gegenübergestellt und über die Möglichkeiten und Grenzen des Technologieeinsatzes reflektiert. Um in Zukunft produktivitätssensitive Augmented-Reality-Assistenzsysteme, die ihre Unterstützung im Hinblick auf den tatsächlich erzielten Effekt permanent anpassen, entwickeln zu können, wird am Ende des Beitrages ein entsprechender Forschungsbedarf abgeleitet.

\section{AR-Assistenzsysteme zur Steigerung von Produktivität}

Die Industrie verändert sich derzeit drastisch. Vor allem IT-getriebene disruptive Veränderungen wirken auf unterschiedlichste Geschäftsfelder und im Besonderen auf die Fertigungsindustrie ein. Dieser Veränderungsprozess und die zugehörigen Technologien werden weitgehend im Begriff Industrie 4.0 zusammengefasst (acatech - Deutsche Akademie der Technikwissenschaften e. V. 2020). Einerseits wollen produzierende Unternehmen auch in Zukunft für hochqualifizierte Arbeitende attraktiv bleiben und werden daher mensch-zentrierte IKT-Ansätze verfolgen müssen (Stocker et al. 2014). Andererseits drängen neuartige Geräte für den Industrie- und vor allem auch für den Consumer-Bereich mit dem Versprechen auf den Markt, die Produktivität mit neuartigen Ansätzen und Lösungen zu steigern.

Hinsichtlich der Gestaltung neuer Informationssysteme haben vor allem Augmented-Reality-Systeme, beflügelt durch die stetige Steigerung ihrer Leistungsfähigkeit, in den letzten Jahren viel Aufmerksamkeit erhalten. Im Reality-Virtuality Continuum definieren Milgram et al. (1995) AR als einen Bereich der Mixed Reality, in dem eine reale Umgebung mit virtuellen Elementen angereichert wird bzw. virtuelle Elemente in eine reale Umgebung eingeblendet werden. Während Anwendende in einer VR Umgebung komplett in diese eintauchen und die reale Umgebung nicht mehr sehen können, können sie in einer AR Umgebung die reale Umwelt sehr wohl optisch wahrnehmen und sehen zusätzlich dazu auch noch virtuelle Objekte im Kontext dieser Umgebung. Damit ergeben sich für Benutzende neue Formen der Wahrnehmung und der Erlebbarkeit der betrachteten Umgebung bzw. der betrachte-

${ }^{1}$ LEGO ${ }^{\circledR}$ ist ein Markenzeichen der LEGO-Firmengruppe. 
ten Objekte. Smart Glasses und AR-Brillen zeichnen sich wesentlich dadurch aus, dass sie von Anwendenden nicht mit den Händen gehalten werden müssen und daher ihre Hände für die eigentliche Tätigkeit freihalten können. Im Gegensatz zu früheren Smart Glasses wie der Google Glass oder der Vuzix M100, die noch über eine vergleichsweise geringe Rechenleistung verfügten, verfügt die HoloLens von Microsoft als aktuelle AR-Brille über ausreichend Rechenleistung auch für anspruchsvollere industrielle Anwendungen.

Über ein reines AR-System hinaus umfasst ein AR-Assistenzsystem auch ein Informationssystem im Hintergrund (Spitzer et al. 2019), welches entsprechende Steuerungs- und Visualisierungssoftware auf dem AR-Gerät nutzt, um Nutzenden Hilfe und Inhalte anzubieten. Für eine spezifische Verwendung eines AR-Systems müssen der darzustellende Inhalt und die Form der Unterstützung für Anwendende definiert, aufbereitet und gestaltet werden. Dem Informationssystem kommt dabei die Aufgabe $\mathrm{zu}$, diese Inhalte vorzuhalten und bei Bedarf entsprechend zur Verfügung zu stellen. Insgesamt bieten AR-Assistenzsysteme die Möglichkeit, das Sichtfeld der benutzenden Person situativ mit relevanten Inhalten $\mathrm{zu}$ überlagern und über eine intuitive Steuerung durch Gesten oder Sprache mit diesen Inhalten zu interagieren.

Am Ende jeder Einführung einer neuen Technologie in einen bestehenden Prozess sollte, unabhängig vom Potential zur Steigerung der Produktivität durch die verschiedenen Systemen, immer eine abschließende Betrachtung von Kosten- und Nutzenaspekten stehen (Rosenberger und Stocker 2017). Dabei stellt vor allem die Identifikation und Auswahl relevanter Faktoren zur Produktivitätsmessung eine Herausforderung dar - umso mehr, wenn nicht nur einfach messbare Größen wie die Bearbeitungszeit herangezogen werden sollen, sondern auch mitarbeiterbezogene Faktoren, die auf die Produktivität einwirken. Im folgenden Abschn. 3 werden daher Faktoren vorgestellt, die auf die persönliche Produktivität einwirken und die potenziell zur Beurteilung der Produktivität im konkreten Beispiel (vgl. Abschn. 4) herangezogen werden können.

\section{Wirkfaktoren auf die Produktivität und ihre Messung}

Zur Identifikation verschiedener, mittels IT messbarer Wirkfaktoren auf die individuelle Produktivität wurde eine systematische Literaturanalyse für die Jahre 2010 bis 2018 durchgeführt, deren Ergebnis detaillierter in Poppe et al. (2019) beschrieben ist. Die meisten untersuchten Arbeiten befassen sich mit der Analyse von aufgezeichneten Daten, um bspw. den aktuellen Zustand einer Person zu bestimmen oder Veränderungen dessen vorherzusagen.

Wirkfaktoren Die in der untersuchten Literatur gefundenen Wirkfaktoren auf die Produktivität sind mehrheitlich die kognitive Performance, z. B. Tsunoda et al. (2017), sowie Stress, z.B. Rodrigues et al. (2018). Mit dem Konstrukt Stress war dabei stets der negative Stress durch zu hohe Arbeitsbelastung gemeint. Andere untersuchte Bereiche waren die Bestimmung oder Vorhersage des Wohlbefindens oder Glücksgefühls von Personen, z.B. Gloor et al. (2018), und die Optimierung der Arbeitsplatzgestaltung, z.B. Schwartz et al. (2016). Bei diesen Arbeiten bestand 


\section{Unternehmen}

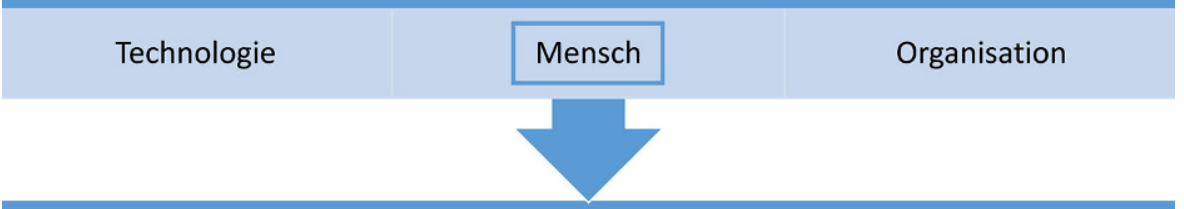

\section{Produktivitätsfaktoren}

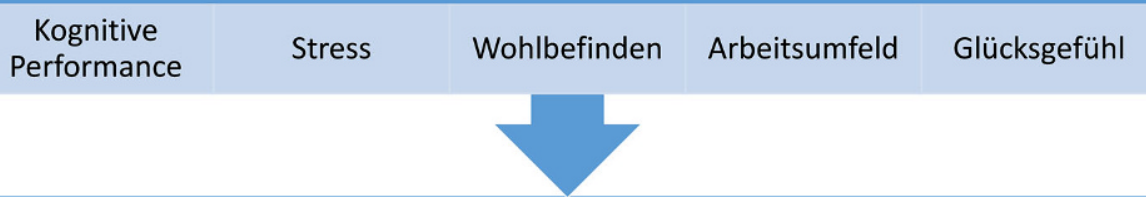

\section{Produktivität $\frac{\text { Realer output }}{\text { Realer Input }}$}

Output-Quantität

Output-Qualität

Abb. 1 Zusammenhang zwischen Arbeitskontext, Wirkfaktoren und Produktivität

die Motivation der Autoren jeweils darin, eine dauerhafte Verbesserung der Bedingungen zu erreichen, damit die Produktivität der arbeitenden Personen langfristig erhalten oder gesteigert werden kann.

Messgrößen und Messinstrumente In der Literaturanalyse wurde auch untersucht, welche Messgrößen bzw. Parameter betrachtet werden und mit welchen Messinstrumenten bzw. Sensoren die jeweiligen Autoren die Wirkfaktoren messen. Es zeigt sich, dass zur Untersuchung der Zustände von Personen oftmals die aus einer Analyse eines Elektrokardiogramms (EKG) gewonnene Herzratenvariabilität (HRV) verwendet wird. Aber auch die physische Aktivität, der elektrische Hautleitwert, die Hauttemperatur und die Auswertung einer Elektroenzephalografie (EEG) wurden unter anderem bereits zur Beurteilung der Produktivität verwendet. Durch die immer kleinere und leistungsfähigere Hardware werden bei diesen Untersuchungen neben Smartphones auch andere kleine, nicht störende Geräte wie Smartbands, Smartwatches oder Stirnbänder zur Datenaufnahme verwendet, welche mit Sensoren bzw. Computerchips ausgestattet sind. Auch tragbare Hardware für EEG-Aufzeichnungen wurde verwendet, die ohne zu stören in Helmen integriert war.

Insgesamt wird deutlich, dass in der Literatur zur Produktivitätsmessung ein breites Spektrum an Wirkfaktoren auf die Produktivität (im Folgenden kürzer als Produktivitätsfaktoren bezeichnet), Messgrößen und Messinstrumenten diskutiert und angewendet wird. Die Produktivitätsfaktoren werden durch den Arbeitskontext im Unternehmen beeinflusst und wirken auf die Produktivität ein. Dieser Zusammenhang wird noch einmal durch Abb. 1 illustriert (die Reihenfolge der Nennung der Produktivitätsfaktoren von links nach rechts ist ohne Bedeutung). 
Ein Unternehmen als soziotechnisches System besteht aus der Perspektive der Arbeitsgestaltung grundlegend aus den eingesetzten Technologien, dem Menschen und den organisationalen Strukturen und Abläufen. Diese wirken auf die Produktivitätsfaktoren ein, die wiederum die Produktivität beeinflussen.

Während die bisher vollzogene Analyse der Wirkfaktoren und ihrer Messung die prinzipiellen Möglichkeiten zur Produktivitätsmessung aufzeigt, gilt es in konkreten Projekten aus diesem Vorrat an Möglichkeiten die in einem Arbeitskontext möglichen und zugleich von Mitarbeitenden für sinnvoll erachteten Wirkfaktoren auszuwählen. Im nächsten Abschn. 4 wird nun exemplarisch ein konkretes Szenario der Anwendung eines AR-Assistenzsystems vorgestellt, anhand dessen weiterführende Überlegungen zur Produktivität unter Einbeziehung prospektiver nutzender Personen angestellt werden.

\section{Workshopszenario: Montage eines Getriebes mit AR-Unterstützung}

Im Folgenden wird die Montage eines Getriebes mit AR-Unterstützung als Anwendungsszenario vorgestellt, welches später von Teilnehmenden in einem Workshop in Bezug auf Produktivitätsfaktoren betrachtet und analysiert wird.

Dieses Szenario wurde im Rahmen des Horizont-2020-Projekts FACTS4WORKERS $^{2}$ entwickelt, in dem IT-Enabler nahtlos in eine flexible Smart Factory Infrastruktur integriert wurden, wobei diese Infrastruktur auf arbeitnehmerzentrierten und datengesteuerten Technologiebausteinen basiert. Spitzer und Ebner (2017) beschreiben in ihrer Arbeit innerhalb des Projekts FACTS4WORKERS ein Problem Based Learning (PBL) Szenario. Mit diesem Lernszenario sollen Anforderungen aus der Praxis der Produktentstehung in das Forschungslabor übertragen und tieferes Wissen über die Schwierigkeiten und Fallstricke in der Produktentstehung gewonnen werden. In diesem konkreten Fall sollten sich Studierende mit Planetenradgetrieben auseinandersetzen, wie sie beispielsweise in Hubschraubern, Arbeitsmaschinen und Fahrradnabenschaltungen eingesetzt werden. In unterschiedlichen Rollen sollten sie ein solches Getriebe entwickeln bzw. aus LEGO ${ }^{\circledR 3}$ Technic Bausteinen zusammenbauen, darauf basierend eine Zusammenbauanleitung erstellen und in weiterer Folge das Planetenradgetriebe (vgl. Abb. 2) neu zusammenbauen.

Im Unterschied zum vorgestellten Lernszenario will das Projekt iDev $40^{4}$ durch eine enge Verzahnung von Entwicklungsprozessen, Logistik und Produktion mit Industrie-4.0-Technologien einen disruptiven Schritt zur Beschleunigung der Markteinführung von (Industrie-)Produkten erreichen. Um zu untersuchen, inwieweit AR-Technologien Produktionsmitarbeitende interaktiv dabei unterstützen können, Produkte aus Einzelteilen zusammenzubauen, wurde dieses Szenario im Projekt iDev40, im Vergleich zu einem konkreten Anwendungsszenario bei einem Industriepartner, als eine taugliche Alternative ausgewählt.

\footnotetext{
2 http://facts4workers.eu/.

3 LEGO $^{\circledR}$ ist ein Markenzeichen der LEGO-Firmengruppe.

4 http://www.idev40.eu/.
} 
Abb. 2 Planetengetriebe, erstellt mit Lego-Bausteinen (Spitzer 2020)

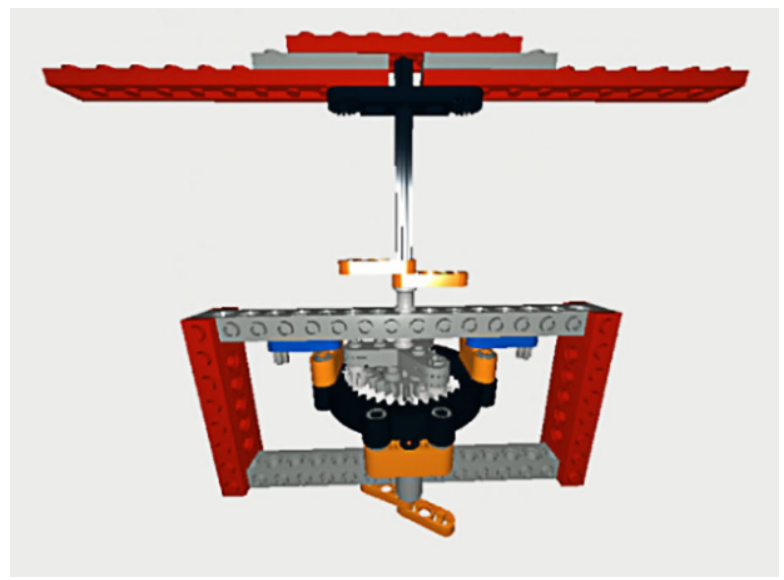

Das oben beschriebene Planetenradgetriebe wurde hier für die im Projekt iDev40 zu untersuchende AR-Unterstützung wiederverwendet. Dafür wurde die bestehende Zusammenbauanleitung als Applikation auf die AR-Brille Microsoft HoloLens portiert und weiterentwickelt. Gesteuert wird diese Applikation über Gesten und ein in das Sichtfeld eingeblendetes Menü (vgl. Abb. 3). Auch wenn in diesem Beitrag speziell die Unterstützung durch eine AR-Brille mit ihren Vor- und Nachteilen bezüglich der Produktivität von Mitarbeitenden untersucht werden soll, so sind im Projekt iDev40 gleichzeitig auch Web-Lösung für PC und Tablet entstanden, die hier aber nicht weiter im Fokus stehen.

Zur Beurteilung der Anwendbarkeit von Produktivitätsmesskonzepten zur Messung des Einflusses von Augmented Reality auf die Produktivität bei Montagearbeiten wurde ein Workshop durchgeführt, der nachfolgend anhand dessen Kontext, Zielen und dem organisatorischen Ablauf näher erläutert wird.

Kontext des Workshops Der Workshop wurde im Begleitprogramm einer Konferenz durchgeführt und die Teilnehmenden konnten sich nach eigenem Interesse für unterschiedliche Workshops anmelden. An diesem Workshop haben 10 Personen aus unterschiedlichen Unternehmen und Unternehmensbereichen teilgenommen.

Ziel des Workshops Die Teilnehmenden des Workshops sollten in einem kreativen Prozess ein Messkonzept erstellen, mit dessen Hilfe Produktivitätsfaktoren aus der Perspektive von betroffenen Personen gemessen werden können. Dabei sollten die Teilnehmenden ihre Erfahrungen im Umgang mit neuen Technologien, in diesem Workshop die HoloLens von Microsoft, reflektieren. Zudem sollten sie die durch diese Technologien verursachten Herausforderungen und Zusatzbelastungen für Anwendende wahrnehmen und benennen. Mit diesen Ergebnissen sollte in einem nächsten Schritt ein realistisches, d.h. technisch machbares und aus Teilnehmendensicht sinnvolles und akzeptables Konzept zur Messung von Produktivitätsfaktoren entwickelt werden. Die für dieses Konzept erforderliche Liste an am Markt verfüg- 
Abb. 3 Zusammenbauanleitung als Microsoft HoloLens Applikation

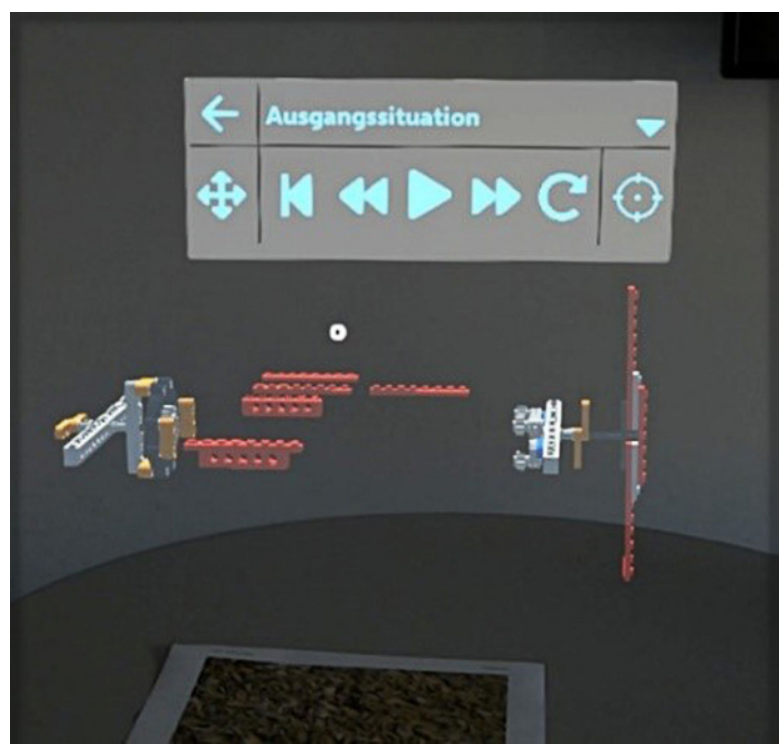

baren Smart Devices (vgl. Abschn. 3: Smartwatch, Smartphone, Smartbands) wurde dazu in einem kurzen Impulsvortrag vorgestellt.

Organisatorischer Ablauf Vor dem eigentlichen Start des Workshops wurde aus den Teilnehmenden eine freiwillige Testperson ausgesucht, welche dann im Workshop, unterstützt durch die AR-Brille, einen ausgewählten Teil der vorbereiteten Zusammenbauanleitung durchführen sollte. Dafür wurde dieser Person in einer kurzen Einführung die Steuerung der AR-Brille bzw. der Zusammenbauanleitung vertraut gemacht. Anschließend sollten im ersten Teil des Workshops dann die anderen Gruppenmitglieder die Testperson beim Zusammenbau des LEGO ${ }^{\circledR}$ Technic Planetenradgetriebes beobachten und deren verbale und nonverbale Äußerungen wahrnehmen und dokumentieren; auch Interpretationen dieser Wahrnehmungen waren erlaubt. Im nächsten Schritt wurden dann diese Beobachtungen und Interpretationen gesammelt, in der Gruppe diskutiert und zusammengefasst. Diese Ergebnisse wurden dann zur kollaborativen Erstellung des Messkonzepts zur Produktivität weiterverwendet. Durch die bei der Erstellung des Produktivitätsmesskonzepts einsetzende Reflexion sollten den Teilnehmenden die Herausforderungen und die physischen Auswirkungen der Verwendung einer AR-Brille bzw. einer neuen Technologie auf die betreffenden Personen noch stärker bewusst werden. Im zweiten Teil des Workshops wurden zunächst in einem Impulsvortrag am Markt verfügbare Smart Devices und deren Möglichkeiten physische Zustände von Menschen zu messen vorgestellt. In der Gruppe wurden auf Basis der Beobachtungen und reflektierten Wahrnehmungen der Testperson die physischen Auswirkungen auf die Testperson analysiert und zusammen mit den zur Verfügung stehenden Messmethoden, mittels Smart Devices, Messkonzepte entwickelt. Mit diesen Messkonzepten sollten Auswirkungen von neuen Technologien als Produktivitätsfaktor erfasst bzw. gemessen werden. Zum 
Abschluss dieses Workshopteils wurden die Ergebnisse final analysiert und kritisch diskutiert.

\section{Produktivitätsmesskonzepte für die AR-unterstützte Getriebemontage}

In dem im vorigen Abschnitt vorgestellten Workshop wurde sowohl eine Auswahl an relevanten Produktivitätsfaktoren im Kontext des vorgestellten AR-unterstützten Montageszenarios als auch ein Konzept für ein Messszenario zur Messung von Wirkfaktoren auf die Produktivität erarbeitet. Diese Ergebnisse wurden zudem auch kritisch beleuchtet und Einwände und kritische Anmerkungen im Hinblick auf Möglichkeiten und Grenzen der Produktivitätsmessung formuliert.

\subsection{Allgemeine Beobachtungen zur Produktivität am Probanden}

Die Testperson wurde spontan und innerhalb kurzer Zeit mit der AR-Brille, der beim Zusammenbau unterstützenden Bedienungsanleitung bzw. der App auf der AR-Brille und der zugehörigen Steuerung der App vertraut gemacht. Sie musste also kurzfristig ein neues Unterstützungssystem verwenden. Diese Herausforderung an die Testperson konnte auch direkt beobachtet werden. Anfangs war sie mehr mit der Steuerung der AR-Brille als mit dem Zusammenbau der vorgesehenen Baugruppe beschäftigt. Allein, dass die Steuerung der AR-Brille anders reagierte als von der Testperson erwartet, führte bei ihr zu beobachtbarem Stress, der sich in hektischen Bewegungen mit dem Kopf und wiederholten Steuerungsgesten mit den Händen ausdrückte. Die Testperson war hier sowohl aus Sicht der unternehmerischen Arbeitsleistung als auch aus ihrer persönlichen Leistungsbilanz - zusätzlicher Stress bei Erbringung gleicher Arbeitsleistung - weniger produktiv. Aus dieser Beobachtung, mit dem Hintergrund der in Abschn. 3 beschriebenen Wirkfaktoren auf die Produktivität und deren Messbarkeit, wurde vor allem zusätzlicher Stress erkannt. Besonders diesem Wirkfaktor auf die Produktivität wurde im Entwurf eines Messszenarios für die Produktivitätsmessung besondere Beachtung geschenkt.

\subsection{Entwurf des Produktivitätsmessszenarios}

Beim Zusammenbau des Planetengetriebes konnten die Teammitglieder an der Testperson immer wieder abrupte Kopfbewegungen beobachten. Nachfolgend hat sich herausgestellt, dass sie das Steuerungsmenü der Microsoft HoloLens Applikation aus dem Sichtfeld verlor und versucht hat, dieses wiederzufinden. Basierend auf dieser Beobachtung und der Rückmeldung der Testperson haben die Workshopteilnehmenden in dieser Situation eine spezifische Stressbelastung interpretiert bzw. identifiziert.

Aus den im beobachteten AR-Szenario abgeleiteten Produktivitätsfaktoren wurde von den Workshopteilnehmenden ein spezifisches, dafür angepasstes Messszenario entworfen. Dieses Messszenario wird hier strukturiert mit Ausgangssituation, Pro- 
blemstellung, Herausforderung, Lösungsansatz und weiterführendem Nutzen vorgestellt:

Ausgangssituation Anwendende von AR-Applikationen könnten das Steuerungsmenü der AR-Applikation aus den Augen verlieren und damit auch die Kontrolle über die AR-Applikation verlieren.

Problemstellung Wenn Anwendende die Kontrolle über die Applikation verlieren führt das zu situativem Stress bzw. werden Mitarbeitende diese neue Technologie langfristig ablehnen.

Herausforderung Es soll erkannt werden, wenn Anwendende die Kontrolle über die AR-Applikation verloren haben.

Evaluierungs-, bzw. Lösungsansatz Die Beobachtung hat gezeigt, dass wenn Testpersonen die Kontrolle über die AR-Applikation verlieren bzw. das integrierte Steuerungsmenü aus den Augen verlieren, dann versuchen das Steuerungsmenü außerhalb das aktuellen Sichtfeldes wiederzufinden. Dies zeigt sich mit abrupten Kopfbewegungen. Die auftretenden Reaktionen bzw. Auswirkungen auf die Physis der Anwendenden sollen folgendermaßen erfasst werden:

- Beschleunigungssensor, um abrupte Kopfbewegungen über Sensoren am Kopf bzw. an oder in der AR-Brille zu messen,

- Smartwatch, um die Herzratenvariabilität als Indikator für Stress zu messen,

- Eyetracking, um hektische Augenbewegungen als Indikator für Stress zu messen,

- Mobiltelefon, um den verbalen Ausdruck der Unzufriedenheit (Fluchen, Beschwerden) zu erfassen.

Weiterführender Nutzen Mit dieser Methode kann in der Einführungsphase von augmentierten Zusammenbauanleitungen erkannt werden, wann und wie oft Anwendende die Kontrolle über die AR-Applikation verloren haben. Die Anzahl der Kontrollverlustereignisse kann dann als ein Indikator für zusätzliche Belastungen bzw. Stress bei Mitarbeitenden herangezogen werden, der sich negativ auf die Produktivität auswirken kann. Die Erkennung des Kontrollverlustes in der AR-Applikation kann gezielt auch dafür verwendet werden, das augmentierte Sichtfeld im Ereignisfall neu auf das Sichtfeld der Anwendenden anzupassen.

\subsection{Kritische Diskussion der Produktivitätsmessung}

Insgesamt hat sich durch die Diskussionen im Rahmen des Workshops gezeigt, dass die Teilnehmenden einer permanenten Produktivitätsmessung mit Sensordaten sehr skeptisch gegenüber eingestellt waren, auch wenn dies nicht immer explizit durch Wortbeiträge geäußert wurde. Wurde hingegen die Produktivitätsmessung mit Sensoren als vorübergehende Maßnahme diskutiert und unter der Voraussetzung, dass die Ziele transparent dargelegt werden und bestehende Datenschutzgesetze und ethische Grundsätze beachtet werden, zeigten sich die Teilnehmenden aufgeschlossener. Die große Reserviertheit und Zurückhaltung der Teilnehmenden wurde jedoch erst 
dann überwunden, als die Produktivitätsmessung von den Moderatoren auch als mögliches Instrument vorgestellt wurde, das den Mitarbeitenden, etwa bei der Evaluierung einer neuen Technologie und zur ergonomischen Gestaltung der Arbeit, zu Gute kommt.

Hieraus kann in Bezug auf die Produktivitätsmessung gefolgert werden, dass erstens eine Messung nur vorübergehend und zum Wohle der Mitarbeitenden (z. B. Erhöhung der Ergonomie oder Vereinfachung der Arbeit) akzeptiert ist. Zweitens kann in Bezug auf die Produktivitätsmessung gefolgert werden, dass eine permanente sensorische Erfassung nur als persönliches Feedback akzeptiert ist, bzw. nur zum Selbstmanagement genutzt werden soll - etwa um Schwankungen der Arbeitsleistung oder Schulungsbedarfe zu erkennen. In diesem Sinne kann die sensorische Erfassung von Produktivitätsfaktoren dazu eingesetzt werden, dass Mitarbeitende ihre Arbeitsweisen und die von ihnen eingesetzten Werkzeuge reflektieren und kontinuierlich verbessern. Damit wird implizit das ökonomische Minimalprinzip verfolgt, das bei einem festgelegten Arbeits-Output die erforderliche persönliche InputLeistung als variable Größe minimiert. Es wäre hier auch denkbar, dass das Maximalprinzip angewendet wird, d.h. bei gleichbleibender, individuell als maximal festgelegter Leistung der Erfolg als variable Größe maximiert wird.

Weiter zeigte sich, dass weitaus mehr Potenzial darin gesehen wird, nicht die Produktivität, sondern andere, sie teils indirekt beeinflussende Faktoren zu messen. Auf der Basis des Feedbacks vom Workshop war insgesamt ersichtlich, dass es aus der Sicht der Technologienutzenden am wichtigsten ist, zu beobachten, wie sich die Arbeit verändert und wie neue Technologien auf die Mitarbeitenden wirken, also ob sie bspw. eine konzentriertere Arbeit befördern oder zu mehr Ablenkung führen, ob eine Zunahme von Stress oder ein entspanntes Arbeiten feststellbar ist und ob die Technologien als Bereicherung der Arbeit empfunden oder als Bevormundung erlebt werden.

\section{Fazit und Ausblick}

Das hier vorgestellte Ergebnis aus dem Workshop ist nicht unabhängig von dem gewählten Ausgangsszenario, der AR-Technologie, sowie der Teilnehmenden und deren Kompetenzen und Erfahrungen zu betrachten und kann sich in einem anderen Kontext von den hier vorgestellten Ergebnissen durchaus auch unterscheiden. Bezugnehmend auf das Ausgangsszenario, basierend auf einem LEGO ${ }^{\circledR}$ TechnicPlantenradgetriebe, und der erfolgreichen Einführung von Innovationen in Unternehmen soll an dieser Stelle noch einmal darauf hingewiesen werden, dass in der Problemanalyse und Lösungsfindung alle Bereiche aus Mensch, Technologie und Organisation (Ulich 2005) berücksichtigt werden sollen. Allzu oft stehen technikzentrierte Ansätze bzw. vorausgewählte Technologien im Vordergrund und verstellen dabei die Sicht auf andere Lösungsansätze, z. B. aus dem organisatorischen, nicht technischen Bereich (Stocker et al. 2014).

Auch aus dem spezifischen Aufbau des Workshops und den daraus erarbeiteten Ergebnissen lassen sich allgemeine Erkenntnisse ableiten bzw. bestätigen. Für eine erfolgreiche Einführung einer Innovation in einem Unternehmen ist es unbedingt 
notwendig, betroffene Personen frühzeitig in diesen Prozess miteinzubeziehen und deren Feedback, Bedenken und Vorschläge zu berücksichtigen. Das betrifft einerseits die Gestaltung und die Bedienung des Assistenzsystems, andererseits auch dessen Evaluierung. Nur so können sowohl aus unternehmerischer, aber vor allem auch aus individueller Sicht Anforderungen, Wünsche, Bedürfnisse und Bedenken, aber auch indirekte Mehraufwände erkannt und berücksichtigt werden.

Im Rahmen der zukünftigen Forschung wäre zu überprüfen, inwieweit die Verlässlichkeit und Tauglichkeit der hier beschriebenen Messkonzepte gegeben ist und wie diese im Vergleich zu spezialisierten, kostenintensiven Messsystemen zur Bewertung der Produktivitätsfaktoren abschneiden. Speziell für die Weiterentwicklung von AR-Assistenzsystemen muss, wie sich bspw. auch in der Beobachtung der Testperson im Workshop gezeigt hat, der Steuerung dieser Anwendungen besonderes Augenmerk geschenkt werden. So muss es in einer AR-Umgebung möglich sein, so weit in diese neue erweiterte Welt einzutauchen, dass die reale und virtuelle Umgebung sowie die Interaktion mit dieser, nahtlos ineinander übergehen und diese Übergänge von Anwendenden beinahe nicht mehr wahrgenommen werden. Fallen diese derzeit noch beobachtbaren Ablenkungen und Zusatzbelastungen weg, dann werden aus Sicht der Mitarbeitenden zukünftig sowohl Akzeptanz als auch eine Steigerung der Produktivität beobachtbar sein. Im Projekt iDev40 wird das Format des Workshops, das hier noch auf der Basis des vorgestellten Montageszenarios beruht, weiterentwickelt. Auf dieser Basis sollen dort sozio-technische Aspekte identifiziert werden, die für eine erfolgreiche Einführung neuer Assistenzsysteme in Betrieben erforderlich sind.

Danksagung The project iDev40 has received funding from the Electronic Component Systems for European Leadership Joint Undertaking under grant agreement No 783163. This Joint Undertaking receives support from the European Union's Horizon 2020 research and innovation programme and Germany, Austria, France, Czech Republic, Netherlands, Belgium, Spain, Greece, Sweden, Italy, Ireland, Poland, Hungary, Portugal, Denmark, Finland, Luxembourg, Norway, Turkey.

Virtual Vehicle Research GmbH has received funding within COMET Competence Centers for Excellent Technologies from the Austrian Federal Ministry for Climate Action, the Austrian Federal Ministry for Digital and Economic Affairs, the Province of Styria (Dept. 12) and the Styrian Business Promotion Agency (SFG). The Austrian Research Promotion Agency (FFG) has been authorised for the programme management.

Funding Open Access funding provided by Projekt DEAL.

Open Access Dieser Artikel wird unter der Creative Commons Namensnennung 4.0 International Lizenz veröffentlicht, welche die Nutzung, Vervielfältigung, Bearbeitung, Verbreitung und Wiedergabe in jeglichem Medium und Format erlaubt, sofern Sie den/die ursprünglichen Autor(en) und die Quelle ordnungsgemäß nennen, einen Link zur Creative Commons Lizenz beifügen und angeben, ob Änderungen vorgenommen wurden.

Die in diesem Artikel enthaltenen Bilder und sonstiges Drittmaterial unterliegen ebenfalls der genannten Creative Commons Lizenz, sofern sich aus der Abbildungslegende nichts anderes ergibt. Sofern das betreffende Material nicht unter der genannten Creative Commons Lizenz steht und die betreffende Handlung nicht nach gesetzlichen Vorschriften erlaubt ist, ist für die oben aufgeführten Weiterverwendungen des Materials die Einwilligung des jeweiligen Rechteinhabers einzuholen.

Weitere Details zur Lizenz entnehmen Sie bitte der Lizenzinformation auf http://creativecommons.org/ licenses/by/4.0/deed.de. 


\section{Literatur}

acatech - Deutsche Akademie der Technikwissenschaften e.V (2020) Industrie 4.0 - acatech. Auf dem Weg ins vierte industrielle Zeitalter. https://www.acatech.de/projekt/industrie-4-0/. Zugegriffen: 3. März 2020

Drucker PF (1999) Knowledge-worker productivity: the biggest challenge. Calif Manage Rev 41(2):79-94. https://doi.org/10.2307/41165987

Gloor PA, Colladon, Fronzetti A, Grippa F, Budner P, Eirich J (2018) Aristotle said "happiness is a state of activity"-predicting mood through body sensing with smartwatches. J Syst Sci Syst Eng 27(5):586-612. https://doi.org/10.1007/s11518-018-5383-7

Milgram P, Takemura H, Utsumi A, Kishino F (1995) Augmented reality: a class of displays on the realityvirtuality continuum. In: Proceedings Volume 2351, Telemanipulator and Telepresence Technologies. Photonics for Industrial Applications, 1994, Boston, MA, United States. https://doi.org/10.1117/12. 197321

Poppe M, Lambusch F, Fellmann M, Lichtwark M (2019) IT-unterstützte Datenerfassung zur Ableitung persönlicher Produktivitätsfaktoren: eine systematische Literaturanalyse. In: Mensch und Computer 2019 - Workshopband (https://dl.gi.de/handle/20.500.12116/25162.)

Rodrigues S, Paiva JS, Dias D, Aleixo M, Filipe RM, Cunha JPS (2018) Cognitive impact and psychophysiological effects of stress using a biomonitoring platform. Int J Environ Res Public Health. https:// doi.org/10.3390/ijerph15061080

Rosenberger M, Stocker A (2017) Eine Vorgehensweise zur Unterstützung der Einführung von Industrie4.0-Technologien

Scharnhorst J (2012) Burnout. Präventionsstrategien und Handlungsoptionen für Unternehmen. Haufe Lexware, Freiburg · München

Schwartz B, Kapellusch JM, Schrempf A, Probst K, Haller M, Baca A (2016) Effect of a novel two-desk sitto-stand workplace (ACTIVE OFFICE) on sitting time, performance and physiological parameters: protocol for a randomized control trial. BMC Public Health. https://doi.org/10.1186/s12889-016$3271-\mathrm{y}$

Spitzer M (2020) vo3xel/LEGO-planetary-gear: manuals. Zenodo, Genève, Switzerland. https://doi.org/ 10.5281 /zenodo. 3696772

Spitzer M, Ebner M (2017) Project based learning: from the idea to a finished LEGO $^{\circledR}$ technic artifact, assembled by using smart glasses. In: Association for the Advancement of Computing in Education (AACE). (Hrsg) Proceedings of EdMedia: World Conference on Educational Media and Technology 2017, S 196-209

Spitzer M, Gsellmann I, Hebenstreit MD, Ebner M (2019) A research agenda to deploy technology enhanced learning with augmented reality in industry. Gesellschaft für Informatik e. V., Bonn. https://doi. org/10.18420/muc2019-ws-300-05

Stocker A, Brandl P, Michalczuk R, Rosenberger M (2014) Mensch-zentrierte IKT-Lösungen in einer Smart Factory. Elektrotech Inftech 131(7):207-211. https://doi.org/10.1007/s00502-014-0215-z

Tsunoda K, Chiba A, Yoshida K, Watanabe T, Mizuno O (2017) Predicting changes in cognitive performance using heart rate variability. In: Institute of Electronics, Information and Communication, Engineers, IEICE (Hrsg) IEICE Transactions on Information and Systems, E100D, S 2411-2419

Ulich E (2005) Arbeitspsychologie, 6. Aufl. vdf Hochschulverl. an der ETH, Zürich (http://deposit.dnb.de/ cgi-bin/dokserv?id=2646503\&prov=M\&dok_var=1\&dok_ext=htm.)

Wöhe G, Döring U, Brösel G (2013) Einführung in die allgemeine Betriebswirtschaftslehre, 25. Aufl. Vahlen, München 